\title{
Visual feedback training in young karate athletes
}

\author{
Stefano Vando ${ }^{1}$ \\ Monoem Haddad ${ }^{2}$ \\ Daniele Masala ${ }^{1}$ \\ Lavinia Falese ${ }^{1}$ \\ Johnny Padulo 3,4
}

1 Department of Humanities, Social and Health Science, University of Cassino and Southern Lazio, Italy

2 Sport Science Program, College of Arts and Sciences, Qatar University, Doha, Qatar

3 Sport Science, University e-Campus, Novedrate, Italy

4 Tunisian Research Laboratory "Sports Performance Optimization" National Center of Medicine and Science in Sport, Tunis, Tunisia

\section{Corresponding author:}

Johnny Padulo

Sport Science, University e-Campus, Novedrate

Via Isimbardi, 10

22060 Novedrate, Italy

E-mail: sportcinetic@gmail.com

\section{Summary}

Background: the purpose of this study was to examine the effect of a Visual Feedback Training (VFT) of the centre of pressure (COP) on postural sway in young karate athletes. Methods: 38 young male karate athletes were recruited and randomly in 2 groups: experimental group (SG; $n=19$, age $=16.54 \pm$ $2.00 \mathrm{yrs}$ ) and the control group (CG; $n=19$, age 16.45 $\pm 1.53 \mathrm{yrs}$ ). The SG performed a battery test of VFT on stabilometric platform screening in a monitor of COP in a real time. During 10-min of the test, athlete tries to bring his own body to the centre of COP. CG followed the same procedure of SG without receiving any feedback of their COP. Results: ANOVA revealed an interaction training for type $\times F_{(1,360)}=78.892$ at $p<0.001$ $\left(\eta^{2}=33.178\right)$ and for training $x$ time $F_{(1,36)}=7.479$ with $p<0.010\left(\eta^{2}=13.432\right)$. The results showed that VFT improved COP in SG of the $83 \%(p<0.0001)$ and decreased of the $26 \%$ in the CG $(p<0.001)$. Conclusions: performing VFT improve stability and balance control and therefore greater ability to acquire motor control of the body in young karate athletes.

KEY WORDS: balance, central pattern generator, martial artists, motor control, stabilometric.

\section{Introduction}

Postural sway is a fundamental human physiology phenomenon where the human body constantly displaces and corrects the position of the centre of gravity within the base of body support in order to maintain balance ${ }^{1}$. Since most other body actions are based on postural balance. Thus effectively controlling postural sway into a balance range plays an essential role in human's normal life, and then this essential role makes postural control become an attractive object of various investigations in wide research areas of exercise physiology, experimental psychology, cognitive science and clinical medicine.

Postural control becomes an attractive object of various investigations in wide research areas in young and adult athletes or patients since most other body actions are based on postural balance ${ }^{2,3}$. In general, postural control of normal healthy persons is highly related with the visual information that they have obtained. The scientific findings of the effects of visual feedback on upright postural control are controversial. Some researchers ${ }^{4-7}$ presented significant postural improvement and shown that Visual feedback training (VFT) of postural sway: 1) helps both healthy athletes and patients (with unperturbed stance) to be aware of their postural sway to improve its control, 2) keeps the body close to the vertical, to enlarge the boundaries of the area in which the body can oscillate safely, and 3) reduce left-right difference in weight bearing. There allow small body oscillations close to the vertical to reduce muscle activity, which requires less energy and larger distances between the gravity line and the limits of stability to safer and make recovery from perturbations more successful as shown by Duarte and Zatsiorsky (2002) ${ }^{8}$. Due appears the important of VFT in martial arts such as Karate. In other hand, others reported no enhancement of posture control ${ }^{9,10}$. This controversy inspires researchers to further investigate whether and how the effects of visual feedback on postural control depend on the way the visual feedback is provided.

Recently studies showed that new technology based on Play games "i.e. Nintendo" Wii Balance Board (WBB), can be a valid device to assess postural sway ${ }^{11}$. Indeed the WBB analyse the centre of pressure (COP) in clinical and sport field ${ }^{3}$. Several studies showed improvements in postural sway ${ }^{5}$ symmetry ${ }^{12}$ and dynamic ability ${ }^{13}$ providing visual Feedback. Indeed, the use of WBB might be a useful and low cost device to assess VFT ${ }^{14}$. To date, to our best knowl- 
edge, there is no report on the additive value of VF to balance training paradigms for young martial artists. Therefore, the aim of the present study was to examine the effects of balance training when standing with visual feedback on stance in young karate athletes. We hypothesized that this type of training will enhance stability and balance control.

\section{Material and methods}

\section{Subjects}

Thirty-eight young male karate athletes (means \pm SD: age $16.42 \pm 2.76 \mathrm{yrs}$, height $164 \pm 6.73 \mathrm{~cm}$, body mass $54.19 \pm 7.15 \mathrm{~kg}$ ) volunteered to participate in this study. All participants was randomly ${ }^{15}$ in 2 groups: Experimental group (SG; $n=19$, age $16.54 \pm 2.00 \mathrm{yrs}$ ), and Control group (CG; $n=19$, age $16.45 \pm 1.53$ yrs). Both groups had at least 3.8 years of karate training background with two/three karate training session per-week (total 3/4 hours per week). None of the subjects ever underwent any endurance strenuous activity or resistance training outside of their normal training program. The study conformed to the Declaration of Helsinki 1964 and was conducted after approval from the local Ethics Committee wich followed the ethical standard of the Muscles, Ligaments and Tendons Journal ${ }^{16}$. The procedures, risks and goals were explained to the participants tutor and written parental consent was obtained prior to participation in addition to the subjects' consent.

\section{Experimental set-up}

The procedure was performed on 3 steps with one day of recovery in-between. In the first and last step all participants was assessed in two occasions (Intraclass Correlation Coefficient) ${ }^{17}$ on a stabilometric platform with CoreMeter ${ }^{\mathrm{TM}}$ software to analyze the centre of the pressure (COP) from the point of origin the Cartesian plane ${ }^{3}$. The second step, SG performed a battery test of VFT on stabilometric platform that screen in a monitor the COP in real time (Fig. 1) during a total duration of $10 \mathrm{~min}$. Athletes' are asked to bring his own body to the COP. CG performed the same battery test as SG without receiving any feedback of their COP.

This battery test of postural stability lasted 20-s during which eyes open on Nintendo ${ }^{\mathrm{TM}}$ Wii Balance Board (WBB). The WBB, validated by Clark et al., ${ }^{11}$ contains 4 micro foil-type strain gauge transducers that are located in each of the 4 corners of the board with Sampling rate at $100-\mathrm{Hz}$. The WBB was interfaced with a laptop computer using custom-written software CoreMeter ${ }^{\mathrm{TM}} 0.9$ (Latina, Italy) and calibrated by placing a variety of know loads at different positions on the WBB. Random order is respected during the protocol procedure (Latin square design) with 1 minute of recovery between trials. During each trial, young karate athletes were asked to keep their hands

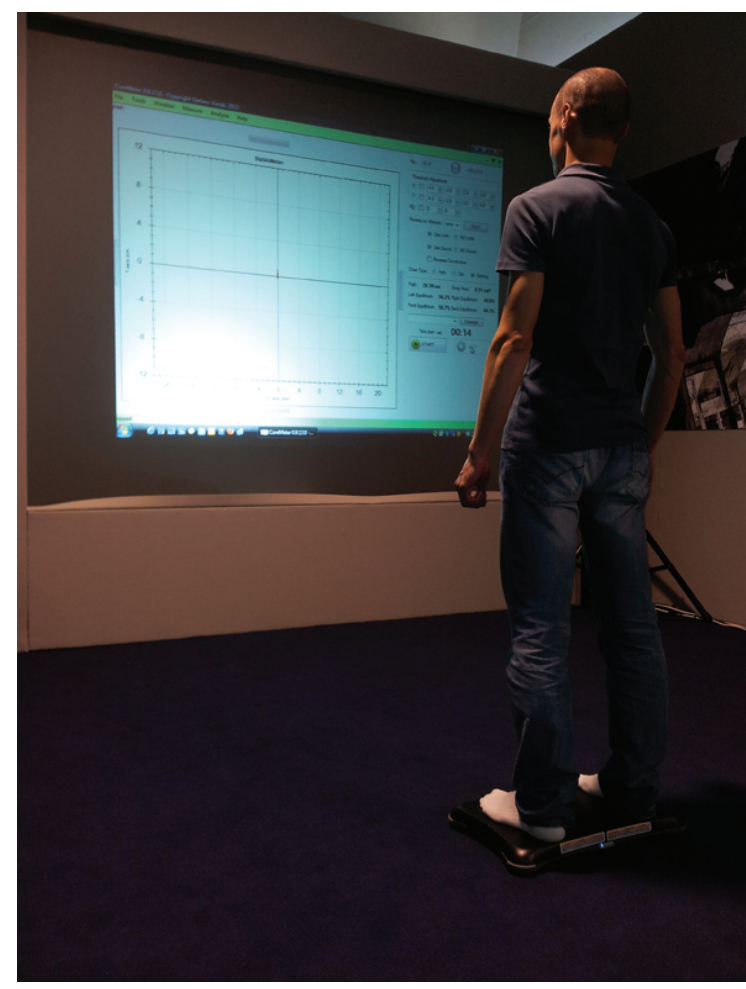

Figure 1. Visual feedback training.

placed on their hips and to remain as still as possible for the duration of the trial.

The WBB is a peripheral Bluetooth device that requires pairing with the personal computer to establish communications. Once paired successfully, the device can be accessed through the standard Bluetooth stack. The device can be interrogated at any time to read the current settings from the four strain-gauge sensors on the board, which are delivered as 16-bit integers. By taking into account the position of the sensors and the values recorded, the position of the COP can be easily calculated. The WBB sensors have an internal fixed sampling rate, which we determined to be $100-\mathrm{Hz}$. Raw calibration data and raw sensor values are stored in a relational database on the local machine. This allows for flexible post -test processing of data. A report generation tool analyzes collected data from the database and produces summary reports. The outcome measure used in this study was total COP. Therefore total COP was chosen as the primary outcome measure because it is known to be a reliable and valid measure of standing balance.

\section{Visual Feedback Training}

Each young karate athlete performed a standing position on WBB during 10-minutes to familiarize with the position. Then, he positioned on WBB for experimental procedure and asked to stand still on the platform and try to be the most central without outside visual signal by researcher. At the last step, SG performed during 10 min - Visual Feedback Training (Fig. 1) on 
stabilometric platform (that screen in a monitor the COP in real time) trying to bring his own body to COP.

\section{Statistical Analysis}

Normality of the distribution for outcome measures was tested using the Shapiro-Wilk normality test. The variables studied: DCOP were analysed using a separated two-way ANOVA with repeated measures and Bonferroni's correction ${ }^{18}$. For testing the repeatability of the measure, we performed an Intra-class Correlation Coefficient (ICC) within COP before the start of this study as a standard quality assessment procedure. The within factor was the time with two levels (pre and post- feedback effect) and the between factor was the training with two levels (SG and $C G$ ). The $t$-student test for independent samples was used to detect any initial differences between groups ${ }^{19}$. The probability level of statistical significant was set at $p<$ 0.05 in all comparisons. Data were analyzed using XLSTAT 12.3.01 (Addinsoft, SARL, New York) statistical software package. Descriptive statistics were expressed as mean \pm SD.

\section{Results}

The reliability of the measures showed an ICC of 0.909 of COP. ANOVA revealed an interaction training for type $\times F_{(1,36)}=78.892$ at $p<0.001\left(\eta^{2}=\right.$ 33.178) and for training $\times$ time $F_{(1,36)}=7.479$ with $p<$ $0.010\left(\eta^{2}=13.432\right)$. The results showed that VFT improved DCOP in SG of the $83 \%(p<0.0001)$ and decreased of the $26 \%$ in the CG $(p<0.001)$ Figure 2 .

\section{Discussion}

The present study is the first to show the effect of VFT (in a real time) of the COP on the stance and stability in young karate athletes. Higher improvement of COP was resulted by the VFT. Main Finding of this study confirmed the hypothesis and shown that the

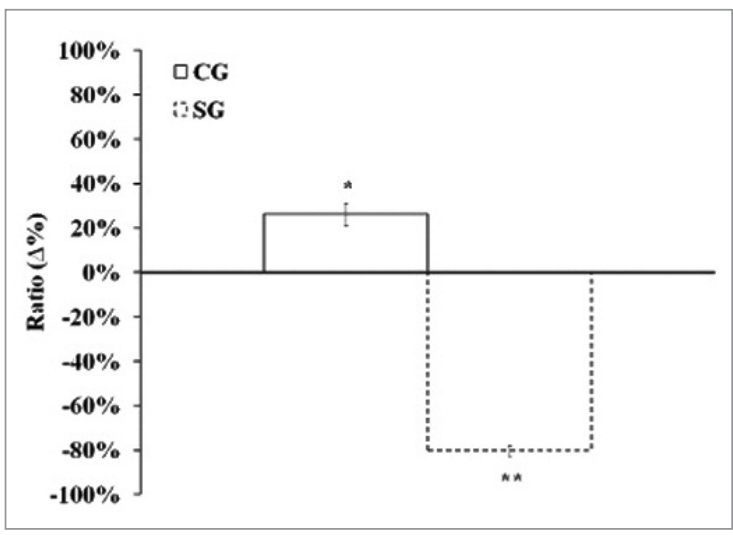

Figure 2. Effect of visual feed-back training respect to baseline conditions in both groups (experimental and the control groups). ${ }^{*} p<0.001-{ }^{* *} p<0.0001$.
VFT improve the sensibility of the postural sway. Results of the present study confirmed the positive effects of visual feedback on upright postural control as shown by many other researchers ${ }^{4-8}$.

Indeed, stabilometric analysis the athletes revealed that a higher central in SG than in CG. This result is consistence with the study of Vando et al. $^{3}$ In another study, Vando et al. ${ }^{7}$ showed an improving on the balance in older when the stabilometric device is related to virtual reality. Moreover, the balance performance in Karate is useful when the subject is on one leg in a short time especially that several karate training are made on one leg with continuous jump ${ }^{20}$.

However, the results of the present study disagreed with those of other researchers who reported no enhancement of posture control ${ }^{9}, 10$. This controversy may be caused by the difference of the providing way of the visual feedback. The present study support the validity and the use of the new technology based on Play games "i.e. Nintendo $\mathrm{Wii}^{\mathrm{TM}}$ " WBB ${ }^{11}$. The developments of this Novel technological allow for integrating an athlete's own movements in virtual environments, alongside other elements borrowed from the gaming industry, such as real-time $3 D$ rendering, avatars, and score-keeping. Balance exercises are often included in game consoles (such as Nintendo $\mathrm{Wii}^{\mathrm{TM}}$ as used in the present study). The methodological approach used to assess postural sway might be useful to improve the proprioception sensibility with simple play device ${ }^{21}$ in martial artists with special reference to young karate athletes. However, further study still necessary to clarify the origin of this effect: central neurons or central pattern generator ${ }^{13}$. In conclusion, one session of VFT appears effective to improve the centrality of the COP in young karate athletes. Based on the scientific literature aimed on balance training, greater ability to acquire motor control of the body is shown with young athlete. Therefore, it is desirable to use this method in young martial artists.

\section{Competing interests}

The authors declare no conflict of interest related to the present manuscript.

\section{Funding}

No funding was received for this article.

\section{Authors Contribution}

All authors contributed equally to this manuscript.

\section{References}

1. Pei L, Li H, Fu Y, Yang Y, Li J. Influences of visual feedback indicator scales on human upright postural control. Transactions of the Institute of Measurement and Control. 2013;35:883. 
2. Goble DJ, Coxon JP, Van IA, Geurts M, Doumas M, Wenderoth N, Swinnen SP. Brain activity during ankle proprioceptive stimulation predicts balance performance in young and older adults. J Neurosci. 2011;31(45):16344-16352.

3. Vando S, Filingeri D, Maurino L, Chaabene H, Bianco A, Salernitano G, Foti C, Padulo J. Postural Adaptations in Preadolescent Karate Athletes Due to a one Week Karate Training Camp. J Hum Kinet. 2013;38:45-52.

4. Sackley CM, Lincoln NB. Single blind randomized controlled trial of visual feedback after stroke: effects on stance symmetry and function. Disabil.Rehabil. 1997;19(12):536-546.

5. Shumway-Cook A, Anson D, Haller S. Postural sway biofeedback: its effect on reestablishing stance stability in hemiplegic patients. Arch Phys Med Rehabil. 1988;69(6):395-400.

6. Wannstedt GT, Herman RM. Use of augmented sensory feedback to achieve symmetrical standing. Phys Ther. 1978;58(5): 553-559.

7. Vando S, Unim B, Cassarino SA, Padulo J, Masala D. Effectiveness of perceptual training - proprioceptive feedback in a virtual visual diverse group of healthy subjects: a pilot study. Epidemiology Biostatistics and Pubblic Health. 2013;10(2): e8844-1-e8844-10.

8. Duarte M, Zatsiorsky VM. Effects of body lean and visual information on the equilibrium maintenance during stance. Exp.Brain Res. 2002;146(1):60-69.

9. Di Fabio RP, Badke MB, McEvoy A, Breunig A. Influence of local sensory afference in the calibration of human balance responses. Exp Brain Res. 1990; 80(3):591-599.

10. Geiger RA, Allen JB, O'Keefe J, Hicks RR. Balance and mobility following stroke: effects of physical therapy interventions with and without biofeedback/forceplate training. Phys Ther 2001;81(4):995-1005.

11. Clark RA, Bryant AL, Pua Y, McCrory P, Bennell K, Hunt M. Va- lidity and reliability of the Nintendo Wii Balance Board for assessment of standing balance. Gait Posture. 2010;31(3):307-310.

12. Winstein CJ, Gardner ER, McNeal DR, Barto PS, Nicholson DE. Standing balance training: effect on balance and locomotion in hemiparetic adults. Arch Phys Med Rehabil. 1989; 70(10):755-762.

13. Cheng PT, Wang CM, Chung CY, Chen CL. Effects of visual feedback rhythmic weight-shift training on hemiplegic stroke patients. Clin Rehabil. 2004;18(7):747-753.

14. van den Heuvel MR, van Wegen EE, de Goede CJ, BurgersBots IA, Beek PJ, Daffertshofer A, Kwakkel G. The effects of augmented visual feedback during balance training in Parkinson's disease: study design of a randomized clinical trial. BMC Neurol. 2013;13:137.

15. Schulz KF, Grimes DA. Generation of allocation sequences in randomised trials: chance, not choice. Lancet. 2002;359 (9305):515-519.

16. Padulo J, Oliva F, Frizziero A, Maffulli N. Muscles, Ligaments and Tendons Journal. Basic principles and recommendations in clinical and field science research. MLTJ. 2013;4:250-252.

17. Hopkins WG. Measures of reliability in sports medicine and science. Sports Med. 2000;30(1):1-15.

18. Cohen J. Statistical power analysis for the behavioral sciences. (2nd ed.) Hillsdale, NJ.Erlbaum. 1988.

19. Faul F, Erdfelder E, Lang AG, Buchner A. G*Power 3: a flexible statistical power analysis program for the social, behavioral, and biomedical sciences. Behav Res Methods. 2007;39 (2):175-191.

20. Beneke R, Beyer T, Jachner C, Erasmus J, Hutler M. Energetics of karate kumite. Eur J Appl Physiol. 2004; 92(4-5):518-523.

21. Banister EW. Modeling elite athletic perfromance. In: Green $\mathrm{H}$ McDougal J, Wenger $\mathrm{H}$, eds. Modeling elite athletic performance.Champaign: Human Kinetics. 1991;403-424. 